\title{
Use of platelets in transfusion medicine
}

\author{
DANIEL B. BRUBAKER, DO
}

Platelet transfusions are beneficial to treat or prevent bleeding in the thrombocytopenic patient. They are frequently used in patients with hypoplastic bone marrow, in cardiovascular surgery patients, and those involved in trauma. Because platelets have short survival, large numbers of platelet units are required. Also, platelet transfusions are expensive and not without complications. They can cause alloimmunization, provoke transfusion reactions, or transmit infectious disease, of which hepatitis C (non-A, non-B hepatitis) is of greatest concern. Therefore, documented indications and close monitoring of the transfused platelets are necessary.

The availability of platelet concentrate transfusions in the 1960 s led to a considerable reduction in death due to hemorrhage in leukemia patients. ${ }^{1}$ From 1971 to 1980 , platelet usage increased nearly $600 \%$ as new indications were explored. ${ }^{2}$ However, recent audits have shown that $40 \%$ of all platelet transfusions were used inappropriately. ${ }^{3}$ In 1985 , the American Association of Blood Banks (AABB) Com-

\footnotetext{
Dr Brubaker is assistant professor, UCLA School of Medicine, Los Angeles, and head of transfusion medicine laboratory and diagnostic immunology, department of pathology, Harbor-UCLA Medical Center, County of Los Angeles, Torrance.
}

The views expressed herein are those of the authors and do not necessarily reflect the views of the county of Los Angeles Department of Health and Human Services or the University of California.

Reprint requests to Daniel B. Brubaker, DO, department of pathology, Harbor-UCLA Medical Center, 1000 W Carson St, Torrance, CA 90509. mittee on Hospital Transfusion Practices published guidelines for the improvement of blood transfusion practices. ${ }^{4}$ At the same time, the Joint Commission on Accreditation of Hospitals $^{5}$ mandated an evaluation of the appropriateness of all blood component transfusions that their inspections closely evaluate. More recently, a National Institutes of Health (NIH) Consensus Conference convened to address various issues in platelet therapy. ${ }^{6}$ The wide dissemination that the AABB guidelines and consensus report have received will lead to improved platelet use. However, there is a continuing lack of consensus in many areas of platelet therapy because of a lack of understanding of platelet function.

Platelets are cell fragments with disk-like structures that prevent bleeding. Their function in hemostasis is to adhere to the subendothelial collagen microfibrils during vascular injury, secrete adhesive proteins, activate other platelets to aggregate, and induce the intrinsic coagulation pathway.

Normal adults have 150,000 to 450,000 platelets per cubic millimeter in the circulation. If either the number of platelets is reduced or the function is abnormal, bleeding may result. In vivo platelet function can be assessed by measuring the in vivo bleeding time. Patients are not very compliant with the test, and there is a large coefficient of variation with a normal bleeding time between 2 and 8 minutes; therefore, a standardized test of platelet function is needed. Bleeding, however, does not usually occur unless the bleeding time is twice normal. In general, a platelet count of 50,000/ $\mu \mathrm{L}$ is adequate for hemostasis. Spontaneous hemorrhage does not occur unless the platelet count is less than $10,000 / \mu \mathrm{L}$.

Thrombocytopenic bleeding is unique in that bleeding arises from small vessels and capillaries of the skin and mucosa. The severest 


\begin{tabular}{|c|c|}
\hline \multicolumn{2}{|c|}{$\begin{array}{c}\text { Table } 1 \\
\text { Indications for Platelet Transfusions }\end{array}$} \\
\hline Diagnosis & $\begin{array}{l}\text { Need for } \\
\text { platelet transfusions }\end{array}$ \\
\hline $\begin{array}{l}\text { Thrombocytopenia due to } \\
\text { decreased production: } \\
\text { Leukemia-postinduction of } \\
\text { chemotherapy } \\
\text { Bone marrow transplantation } \\
\text { Aplastic anemia } \\
\text { Myelofibrosis/chronic myelog- } \\
\text { enous leukemia }\end{array}$ & $\begin{array}{l}\text { Useful to treat or pre- } \\
\text { vent bleeding }\end{array}$ \\
\hline $\begin{array}{l}\text { Dilutional thrombocytopenia } \\
\text { (as in massive transfusion) }\end{array}$ & $\begin{array}{c}\text { Needed when platelet } \\
\text { count }<50,000 \text { or in } \\
\text { excessive bleeding }\end{array}$ \\
\hline $\begin{array}{l}\text { Thrombocytopenia due to in- } \\
\text { creased destruction: } \\
\text { Idiopathic thrombocytopenic } \\
\text { purpura } \\
\text { Thrombotic thrombocytopenic } \\
\text { purpura } \\
\text { Hemolytic uremic syndrome }\end{array}$ & $\begin{array}{l}\text { Not indicated unless } \\
\text { bleeding is life- } \\
\text { threatenting }\end{array}$ \\
\hline $\begin{array}{l}\text { Platelet function abnormality } \\
\text { (as in uremia and plasma } \\
\text { cell dyscrasias) }\end{array}$ & $\begin{array}{l}\text { Only after corrected } \\
\text { functional defect }\end{array}$ \\
\hline
\end{tabular}

form of thrombocytopenic bleeding is intracerebral bleeding, which may be rapidly fatal. Factors that exacerbate bleeding in thrombocytopenic patients include a rapid fall in platelet count, fever, sepsis, certain medication such as aspirin, vomiting, uremia, concomitant coagulation factor deficiency, and trauma.

\section{Indications for platelet transfusions}

Platelet transfusions are indicated for bleeding patients with thrombocytopenia due to decreased marrow production (Table 1). A dilemma occurs in the prophylactic use of platelet transfusion. Some authorities ${ }^{6}$ believe that no prophylactic platelet transfusions are indicated, whereas others ${ }^{1}$ give platelets routinely when the patient's count drops below 20,000 / $\mu \mathrm{L}$. This cutoff number originated from the 1960 s and 1970 s when most patients were treated for fever with aspirin. Because acetaminophen is currently used, the suggested level may be lowered to $10,000^{7}$ or even 5000 / $\mu \mathrm{L}$ (Irecommend $10,000 / \mu \mathrm{L}$ ). Prophylactic platelet transfusions have been recommended only to patients with transient myelosuppression such as induction chemotherapy for acute leukemia, but not to those with chronic myelosuppression, that is, aplastic anemia. ${ }^{6}$

Prophylactic platelet transfusions are given to decrease patient morbidity with bleeding episodes. They do not decrease mortality due to bleeding and require twice the number of transfused platelets for therapeutic results. ${ }^{8,9}$ This issue will remain controversial until reliable, well-controlled studies solve the dilemma.

If surgery or invasive procedures such as lumbar puncture or liver biopsy are necessary, platelet transfusions are required to raise the platelet count above $50,000 / \mu \mathrm{L}$. Platelets are not required if the platelet count is above $50,000 / \mu \mathrm{L}$, and the bleeding time is less than twice the upper limit of normal (12 minutes). ${ }^{6}$

Adjunctive therapy may also be beneficial and may decrease the number of platelets transfused. Epsilon aminocaproic acid (Amicar), an antifibrinolytic agent, may decrease spontaneous bleeding. ${ }^{10} \mathrm{~A}$ preparation of conjugated estrogens (Premarin) can also be used in women to suppress menstruation and menorrhagia. Vitamin $\mathrm{K}$ is useful to prevent coagulation factor deficiency, particularly in patients on antibiotics who are not eating. ${ }^{11}$ Bleeding in uremic patients responds to cryoprecipitate or desmopressin acetate (DDAVP), so that the use of platelet transfusions can be eliminated in such cases.

Dilutional thrombocytopenia from massive blood transfusions, as in trauma, does not present a problem for two reasons: (1) microvascular bleeding does not develop; and (2) platelet counts usually do not drop below 50,000/ $\mu \mathrm{L}$. More than 30 units of blood must be transfused before the platelet count drops below $50,000 / \mu \mathrm{L}$, or there must be coexisting disseminated intravascular coagulopathy (DIC). In the majority of patients receiving 10 to 20 units of blood, bleeding problems do not develop; however, a platelet count should be taken after every 12 to 15 units of blood, with platelet transfusions reserved for thrombocytopenic patients and patients with microvascular bleeding. ${ }^{12}$

Patients who have thrombocytopenia due to peripheral destruction, such as in idiopathic (immune) thrombocytopenic purpura (ITP) or 
thrombotic thrombocytopenic purpura (TTP) do not benefit from platelet transfusions. ${ }^{13}$ Transfusion of platelets in TTP accentuates the disease and can cause rapid demise. Because hemolytic uremic syndrome (HUS) is very similar to TTP, results would be similar. Idiopathic thrombocytopenic purpura rapidly destroys platelets because of an autoimmune antibody toward platelets. The only indication for platelet transfusions in ITP and TTP patients is in life-threatening bleeding such as CNS bleed. ${ }^{12}$ Disseminated intravascular coagulation is more controversial. In my opinion, the underlying cause for DIC should be treated and the patient supported with platelet and cryoprecipitate transfusions.

Cardiac surgery presents further problems in the use of platelet transfusions. One study ${ }^{14}$ has shown no correlation between the platelet count and intraoperative or postoperative bleeding in patients undergoing cardiac surgery. However, other studies ${ }^{15,16}$ have shown significant platelet functional defects and significant postoperative bleeding without platelet transfusions. Functional platelet defects result from cardiopulmonary bypass. ${ }^{16}$ The results of yet another trial ${ }^{17}$ suggest that desmopression may cut platelet transfusions in half in patients undergoing cardiopulmonary bypass. However, many cardiovascular surgeons have not experienced these results.

The effect of aspirin therapy in cardiac surgery patients is also controversial. One study ${ }^{18}$ reported that patients undergoing cardiac surgery who took aspirin were not more likely to bleed than those who did not take aspirin. Other studies ${ }^{19}$ showed prolonged, profuse bleeding in these patients. In my experience and that of others, ${ }^{20}$ as well as the experience of several cardiovascular surgeons, platelet transfusions are indicated postoperatively in cardiac surgery patients until proof shows otherwise.

Other platelet function defects are found in uremia and the dysproteinemias, such as Waldenstrom's macroglobulinemia. Platelet transfusions should not be given to uremic patients until after dialysis or to the patient with dysproteinemia until after plasma exchange.

\section{Platelet products, dosage, and administration}

Platelets are prepared and concentrated by two methods. Random donor or unrelated platelet concentrates are prepared at the community blood center or Red Cross from individual units of whole blood. ${ }^{13}$ The platelet concentrates have about $0.5-1.0 \times 10^{11}$ platelets in $50 \pm$ $10 \mathrm{~mL}$ of plasma. These units are frequently combined as 6-, 8-, 10-, or 12-unit pools.

The second platelet product is prepared from blood drawn from single donors connected to an apheresis instrument, such as Fenwal CS3000 , Cobe Spectrum III, or Haemonetics S-30 instruments. The platelets are separated from the rest of the blood elements and concentrated to give approximately $5 \times 10^{11}$ platelets in one bag. Such products are called single donor platelet concentrates, single donor units, or plateletpheresis units, and each is equivalent to eight random donor units. The advantages of this product is one-donor exposure to HLA antigens and only one risk of hepatitis (hepatitis B or hepatitis C [non-A, non-B]) or acquired immunodeficiency syndrome (AIDS) versus ten risks in the random donor units. ${ }^{13}$ The problem is getting enough donors to meet the need.

All platelet products are stored at room temperature and constantly rotated to have good gaseous exchange and prevent aggregates. Currently, they can be stored for 5 days. Platelets should never be stored in a refrigerator. Both platelet products also contain WBCs, usually about $1 \times 10^{9}$.

The number of units of platelet concentrates to be transfused will depend on the starting platelet count and the desired platelet count. One random donor unit is given per $10 \mathrm{~kg}$ of adult body weight. Each unit will raise the platelet count in an adult by 5000 to 10,000 / $\mu \mathrm{L} .{ }^{13}$ A 10 -unit pool should raise the platelet count in an adult to 70,000 to 100,000 above baseline. One single-donor unit should give a similar incremental rise in the platelet count. The survival of transfused platelets is 5 to 9 days; therefore, platelets are given more frequently than RBCs. Patients should not require more than 12 units every 3 to 4 days. 
Those who require platelet transfusion at shorter intervals have increased platelet destruction of their transfused platelets.

It is desirable to give platelets of the same ABO type as the patient's; however, this is not always possible nor is it essential. ABO antigens on platelets are extremely weak; therefore, platelets are not destroyed through natural ABO isohemagglutinins. There is some $\mathrm{RBC}$ contamination in platelet concentrates (usually less then $0.5 \mathrm{~mL}$ packed cell volume) which can be hemolyzed, but because the volume is so small, the patient experiences no adverse effects. However, there is a risk in pediatric patients or women of child-bearing age, because Rh-positive RBCs could sensitize an Rh-negative patient to produce anti-R $\mathrm{h}_{0} \mathrm{D} .^{21}$

This sensitization occurs infrequently in patients with leukemia because they are immunosuppressed by their disease and by chemotherapy. However, I insist on giving group-specific platelets to these two groups of patients. If $\mathrm{Rh}$ positive platelets are given to a child or to a woman of child-bearing age, $\mathrm{Rh}$ immune globulin can be given. One dose will protect against the RBCs in 30 units of Rh-positive platelets. If enough $\mathrm{ABO}$ mismatched platelets are transfused to a patient, a positive direct antiglobulin test may occur, but significant hemolysis is rare. ${ }^{22}$

The requisite number of random donor platelet concentrates are pooled in the blood bank before transfusion. This provides a convenient and safe product for administration. The platelet concentrates can be stored at room temperature for 5 days, but after pooling, they must be administered within 4 hours (Food and Drug Administration requirement). ${ }^{23}$ Single-donor (plateletpheresis) units can be stored in the blood bank and directly transfused.

Platelets must be administered through the standard $170 \mu \mathrm{m}$ blood filter. Fresh platelets tend to aggregate and get trapped in the filter; however, this usually is not a problem because the platelets are stored and rotated so as to prevent aggregation. In fact, stored platelets can be given through a microaggregate filter; however, this procedure is not recommended for routine administration. ${ }^{24}$
Almost any size needle can be used for platelet administration; however, a 19-gauge works best. Pooled platelets have a final volume of 400 to $600 \mathrm{~mL}$, which would take too long to run in with a small needle. Small-bore needles are acceptable for pediatric patients. To transfuse the maximum number of platelets, the bag and the tubing should be rinsed with $0.9 \%$ saline solution. Gravity infusion is the simplest and most common means of administration. If an emergency situation requires intravenous push, Cutter Biologicals, a division of Miles Laboratories, has made an excellent device to push platelets via a $50-\mathrm{mL}$ syringe (22" Y Blood Component Set with automatic check valves and filter, Code 812-51, Cutter Biologicals, Berkeley, Calif). Platelets may also be given with an infusion pump.

The rate of infusion is 5 to $10 \mathrm{~mL}$ per minute, but it may run as fast as tolerated except in small children, in whom a slow infusion rate is desirable ( 1 to $3 \mathrm{~mL}$ per minute). As with all blood infusions, vital signs should be monitored before and after platelet transfusions.

\section{Monitoring platelet tranfusions}

Platelet counts are essential in platelet therapy to assess the outcome. Certainly stoppage of bleeding after platelet transfusion serves as the best indication of outcome. However, patients frequently are not bleeding at the time of platelet transfusion so other methods are required. A pretransfusion (pre-TFN) platelet count and a 1- to 2-hour posttransfusion (postTFN) platelet count is desirable. ${ }^{13,25}$ The corrected count increment (CCI) is used to monitor patients at Harbor-UCLA Medical Center.

\footnotetext{
CCI $=$ Post-TFN - pre-TFN platelet counts/platelet count in bag $\left(\times 10^{11}\right) \times$ $0.6 \times$ body surface area (BSA) $\mathrm{m}^{2}$.
}

The platelet count is obtained from the pooled bag of platelets before transfusion. At Harbor-UCLA, 0.6 is now used as the splenic pooling factor. Indium 111-labeled transfused platelets have been used to compare platelet counts with platelet survival, and it was found 
that this factor must be used at 1- to 2-hour counts, but not 18- to 24-hour counts. As an example, suppose the pre-TFN count is 10,000 / $\mu \mathrm{L}$ and the post-TFN count was $50,000 / \mu \mathrm{L}$. The platelet count in the bag is $8.4 \times 10^{11}$ and the patient's BSA is $1.6 \mathrm{~m}^{2}$, the CCI is:

$$
40,000 / 5.0[8.4 \times 0.6] \times 1.6=12,800 .
$$

If the CCI is less than 7500 , increased destruction of the transfused platelets is occurring. ${ }^{25}$ For convenience, 10 units of randomdonor platelets or one single donor plateletpheresis should give an incremental rise of more than $30,000 / \mu \mathrm{L}$. A smaller rise indicates increased destruction. There are two reasons for poor posttransfusion increments: antibody destruction and pathophysiologic destruction. ${ }^{26}$

Platelets have strong HLA antigens and platelet-specific antigens on their surface. There is a strong correlation between the presence of HLA antibodies and poor transfusedplatelet survival. ${ }^{26}$ Because of the polymorphism of the HLA system, the contaminating lymphocytes present in the platelet concentrate and the large number of donors the patient is exposed to, alloimmunization is a major problem. ${ }^{27}$ Nearly $50 \%$ of leukemia patients and between $80 \%$ and $100 \%$ of aplastic anemia patients become refractory to randomdonor platelet transfusions. ${ }^{25}$ Many of these patients respond to HLA-matched platelets or platelet crossmatch-compatible platelets collected from single donors by apheresis. ${ }^{26}$ Use of platelets filtered through a cotton wool filter (Imugard IG 500 filter, Terumo Corp, Piscataway, NJ) also prevents refractoriness. ${ }^{28}$ More recently, many physicians are using unrelated non-HLA-matched single-donor platelets at the onset of platelet therapy to prevent alloimmunization. The problem is finding enough donors willing to spend 2 hours on an apheresis instrument.

Pathophysiologic causes of poor posttransfusion increments include sepsis, infections, fever, hepatomegaly, splenomegaly, DIC, hepatic disease, dysproteinemias, ITP, collagen vascular disease, acute renal transplant rejec- tion, and intravenous administration of gamma globulins. ${ }^{25}$ All these decrease transfused platelet survival. However, in many of these diseases, it is not known when the transfused platelet counts decrease; in some cases, it may be within 2 hours of transfusion; in others 12 hours; and in still others, 24 to 36 hours. This is an area for further research.

The method to evaluate appropriateness of all transfusions must be an ongoing, comprehensive approach to quality assurance of clinical transfusion practice. The guidelines set in Harbor-UCLA Medical Center include immediate consultation of the pathologist or transfusion laboratory physician with the requesting physician when platelet transfusion is ordered under the following conditions:

- Platelet count is $>100,000 / \mu \mathrm{L}$ and there is no platelet function defect.

- Platelet count in a preoperative patient is $>50,000 / \mu \mathrm{L}$ or the bleeding time is less than 12 minutes.

- Platelet count in a patient with decreased platelet production is $>20,000 / \mu \mathrm{L}$ and the patient is not actively bleeding.

- Patient's tested bleeding time is $>12$ minutes.

- Patient does not have a platelet count at the time of request.

- Patient who has received multiple platelet transfusions does not have a platelet count increment of at least $30,000 / \mu \mathrm{L}$ (adult receiving 10 random donor units).

- Special requests, for example, HLA-matched platelets, white blood cell-depleted platelets.

- Patient has a diagnosis of ITP, TTP, HUS, or DIC.

- If the physician requests $>15$ units or $<4$ units (unless for a neonatal or pediatric patient) of random-donor pooled platelets.

- Patients receiving more than 12 units within 48 hours.

These same guidelines can be used by a hospital transfusion committee to review inappropriate use in the hospital. There are several different approaches to monitoring blood products, and each institution will usually individualize its own approach. However, the criteria as outlined here for platelet transfusions should be uniform. 


\begin{tabular}{|l|}
\hline \multicolumn{1}{|c|}{\begin{tabular}{c}
\multicolumn{1}{c|}{ Table 2 } \\
Infectious Agents Transmitted \\
by Platelet Concentrate Transfusions
\end{tabular}} \\
\hline Human immunodeficient virus (AIDS)* \\
Hepatits-B* \\
Hepatitis C (non-A, non-B hepatitis) \\
Cytomegalic inclusion virus* \\
Bacteria \\
Brucellosis \\
Babesiasis* \\
Filariosis \\
Rocky Mountain spotted fever \\
Malaria* \\
Syphilis* \\
Toxoplasmosis \\
Trypanosomiasis* \\
*Source: Gilcher RO: Adverse effects of transfusion mediated by infec- \\
tious agents. Plasma Ther Transfus Technol 1985;6:81-93. \\
\hline
\end{tabular}

\section{Complications of platelet transfusions}

Because platelet concentrates also contain WBCs, RBCs, and plasma, recipients may become sensitized to platelet, HLA, granulocyte, $\mathrm{RBC}$, and plasma antigens. As previously mentioned, sensitization to HLA or platelet antigens or both leads to failure to respond to random-donor platelet transfusions (alloimmunization). ${ }^{24,25}$

The two most common transfusion reactions are febrile-nonhemolytic transfusion reaction and allergic transfusion reaction. ${ }^{29}$ Febrilenonhemolytic transfusion reaction is characterized by chills, fever, and flulike symptoms. It is caused by WBC antigen-preformed WBC antibody reaction. Acetaminophen prevents or diminishes the symptoms, and premedication before future transfusions usually prevents symptoms. If symptoms persist, WBC-depleted blood products must be given. ${ }^{29}$ At HarborUCLA Medical Center, these products are prepared in the blood bank by use of a celluloseacetate filter (Erypur Filter, Organon-Teknika Corp, Durham, NC) for RBCs and cotton wool filter (Imugard IG 500) for platelets. I find that these filters are the most cost-effective means of removing leukocytes with very little of the technologists' time. The technique is easy to learn and does not require special equipment.

Allergic reactions are characterized by urticaria, pruritus, skin rashes, periorbital edema, or perilaryngeal edema. ${ }^{29}$ Usually, 25 to $50 \mathrm{mg}$ of diphenhydramine hydrochloride (Benadryl) ends the symptoms. More severe reactions such as perilaryngeal edema should be treated with epinephrine and steroids.

Hemolytic transfusion reactions from the contaminating $\mathrm{RBCs}$ in platelet concentrates are usually not noticed. The small quantity of $\mathrm{RBCs}$ in platelet concentrates, usually $<0.5$ $\mathrm{mL} /$ unit or $5 \mathrm{~mL} / 10$ units, makes hemolysis of donor RBCs insignificant. However, as has already been mentioned, passive transfer of donor anti-A or anti-B may cause the development of a positive direct antiglobulin test result and, rarely, hemolysis as well in the recipient.

Volume overload can be another complication of platelet transfusions. Although each platelet concentrate contains 50 to $70 \mathrm{~mL}$, after pooling the volume can be as high as 800 $\mathrm{mL}$. If volume is a problem for the patient, the platelet pool can be concentrated by centrifugation in the blood bank with as much as 400 to $500 \mathrm{~mL}$ of plasma expelled from the bag. One must keep in mind that these platelets must lie flat with no rotation for 1 to 2 hours to resolve the preparation defect.

Lymphocytes in the platelets cause not only febrile transfusion reactions and platelet alloimmunization, but also graft-versus-host $(\mathrm{GVH})$ disease ${ }^{30}$ This occurs in immunodeficient patients (with the exception of AIDS patients). Posttransfusion GVH disease occurs mostly in patients with acute leukemia, Hodgkin's disease, non-Hodgkin's lymphomas, and severe combined immunodeficiency. To prevent $\mathrm{GVH}$ in these patients, RBCs and platelets are irradiated with $3000 \mathrm{rad}$ at HarborUCLA Medical Center.

Any infectious disease that can be transmitted by blood transfusion can be transmitted by platelet concentrates (Table 2). Because platelet transfusions expose the patient to multiple donors, the risk goes up accordingly. Bacterial infection is of foremost concern with platelets because they are stored at room temperature. ${ }^{12}$ For this reason, all blood banks are required by Food and Drug Administration regulations to store platelet concentrates for 5 days. 


\section{Comment}

In this paper, I have attempted to outline an approach to the use of platelets in transfusion. Indications for transfusions should be documented clearly, and monitoring of platelet transfusions is essential. Because transfusion reactions may occur, platelets should be administered the least number of times possible to minimize risks and complications.

1. Hersh EM, Bodey GP, Nies BA, et al: Causes of death in acute leukemia: A ten-year study of 414 patients from 19541963. JAMA 1965;193:99-103.

2, Surgenor DM, Schmitzer SS: The nation's blood resource. A summary report. NIH publication No. 85-2028. Bethesda, Md, National Institutes of Health, March 1985.

3. Simpson MB: Prospective-concurrent audits and medical consultation for platelet transfusions. Transfusion 1987;27:192195.

4. Grindon AJ, Tomasulo PS, Bergin JJ, et al: The hospital transfusion committee: Guidelines for improving practice. JAMA 1985;253:540-543.

5. Joint Commission on Accreditation of Hospitals. Accreditation Manual for Hospitals. Chicago, The Joint Commission, 1985.

6. Consensus Conference: Platelet transfusion therapy. JAMA 1987;257:1777-1780.

7. Aderka D, Praff G, Santo M, et al: Bleeding due to thrombocytopenia in acute leukemias and reevaluation of the prophylactic platelet transfusion policy. Am J Med Sci 1986;291:147-151.

8. Soloman J, Bofenkamp T, Fahey JL, et al: Platelet prophylaxis in acute non-lymphoblastic leukaemia. Lancet 1978;1:267. 9. Murphy S, Litwin S, Herring LM, et al: Indications for platelet transfusion in children with acute leukemia. Am J Hematol 1982;12:347-356.

10. Gardner FH, Helmer RE III: Aminocaproic acid: Use in control of hemorrhage in patients with amegakaryocytic thrombocytopenia. JAMA 1980;243:35-37.

11. Alperin JB: Coagulopathy caused by vitamin K deficiency in critically ill, hospitalized patients. JAMA 1987;258:19161919.

12. Reed RL II, Ciavarell D, Heimbach DM, et al: Prophylactic platelet administration during massive transfusion. A prospective, randomized, double-blind clinical study. Ann Surg 1986;203:40-48.

13. Eisenstaedt: Blood component therapy in the treatment of platelet disorders. Semin Hematol 1986;23:1-17.

14. Simon TL, Akl BF, Murphy W: Controlled trial of routine administration of platelet concentrates in cardiopulmonary bypass surgery. Ann Thorac Surg 1984;37:359-364.
15. Mohr R, Golan M, Martinowitz U, et al: Effect of cardiac operation on platelets. J Thorac Cardiovasc Surg 1986:92:434441.

16. Mezzano D, Aranda E, Urzúa J, et al: Changes in platelet $\alpha$-thromboglobulin, fibrinogen, albumin, 5-hydroxytryptamine, ATP, and ADP during and after surgery with extracorporeal circulation in man. Am J Hematol 1986;22:133-142.

17. Salzman EW, Weinstein MJ, Weintraub RM, et al: Treatment with desmopressin acetate to reduce blood loss after cardiac surgery: A double-blind randomized trial. $N$ Engl J Med 1986;314:1402-1406.

18. Weksler BB, Pett SB, Alonso D, et al: Differential inhibition by aspirin of vascular and platelet prostaglandin synthesis in atherosclerotic patients. $N$ Engl $J$ Med 1983;308:800805 .

19. Chesebro JH, Fuster V, Elveback LR, et al: Trial of combined Warfarin plus dipyridamole or aspirin therapy in prosthetic heart valve replacement: Danger of aspirin compared with dipyridamole. Am J Cardiol 1983;51:1537-1541.

20. Harker LA, Malpass TW, Branson HE, et al: Mechanism of abnormal bleeding in patients undergoing cardiopulmonary bypass: Acquired transient platelet dysfunction associated with selective $\alpha$-granule release. Blood 1980;56:824-834.

21. Goldfinger D, McGinnis MH: Rh-incompatible platelet transfusions-risks and consequences of sensitizing immunosuppressed patients. N Engl J Med 1971;284:942-944.

22. Pierce RN, Reich LM, Mayer K: Hemolysis following platelet transfusions from ABO-incompatible donors. Transfusion 1980;25:60-62.

23. Code of Federal Regulations. 21CFR 640.26. Washington, DC, Office of the Federal Register, 1986, p 127.

24. Snyder EL, Hezzey A, Cooper-Smith M, et al: Effect of microaggregate blood filtration on platelet concentrates in vitro. Transfusion 1981;21:427-434.

25. Brubaker DB, Duke JC, Romine M: Predictive value of enzyme-linked immunoassay platelet crossmatching for transfusion of platelet concentrates to alloimmunized recipients. Am $J$ Hematol 1987;24:375-387.

26. Brubaker DB, Romine M: Relationship of HLA and plateletreactive antibodies in alloimmunized patients refractory to platelet therapy. Am J Hematol 1987;26:341-352.

27. Duquesnoy RJ: Donor selection in platelet transfusion therapy of alloimmunized thrombocytopenic patients, in Greenwalt TJ, Jamieson GA (eds): The Blood Platelet in Transfusion Therapy. New York, AR Liss Inc, 1978, pp 229-243.

28. Brubaker DB, Romine CM: The in vitro evaluation of two filters (Erypur and Imugard IG 500) for white cell-poor platelet concentrates. Transfusion 1988;28:383-385.

29. Brubaker DB: Immunologically mediated immediate adverse effects of blood transfusions (allergic, febrile nonhemolytic, and noncardiogenic pulmonary edema).Plasma Ther Transfus Technol 1985;6:19-31.

30. Brubaker DB: Transfusion-associated graft-versus-host disease. Human Pathology 1986;17:1085-1088. 